\title{
Authors' reply to Candido-dos-Reis
}

\author{
Dirk Timmerman professor in obstetrics and gynaecology ${ }^{12}$, Jeroen Kaijser research fellow ${ }^{12}$, Tom \\ Bourne professor ${ }^{123}$, Ben Van Calster professor ${ }^{1}$
}

${ }^{1}$ Department of Development and Regeneration, KU Leuven, 3000 Leuven, Belgium; ${ }^{2}$ Department of Obstetrics and Gynaecology, University Hospitals Leuven, 3000 Leuven, Belgium; ${ }^{3}$ Queen Charlotte's and Chelsea Hospital, Imperial College, London, UK

We appreciate Candido-dos-Reis's feedback and agree that the variable "oncology centre," which we defined as "tertiary referral centres with a specific gynaecology oncology unit," is unconventional. ${ }^{2} \mathrm{We}$ anticipated that it would raise some questions. The variable was included because it is a predictor of prior risk of malignancy and because it is still predictive after controlling for the other eight patient specific predictor variables. It is the weakest of the nine ADNEX predictor variables, suggesting that patient specific predictors explain most but not all of the differences in outcomes between oncology centres and other hospitals. In practice this variable is easy to use, because clinicians have to decide only once in what type of centre they work.

The prevalence of adnexal masses is much higher in ultrasound units linked to specialised gynaecological oncology centres than in regional hospitals. This is because patients with more suspicious looking masses are referred for assessment and treatment in specialised centres. We agree that symptoms may add important information and that women with symptoms suspicious of ovarian cancer are more likely to be referred to cancer centres. However, the IOTA study started in 1999 and indices to report symptoms were developed more recently, so we don't know whether patients in oncology centres present with different symptoms and whether the presence or absence of certain symptoms could replace the variable "oncology centre." We are examining symptoms as part of phase 5 of our study and should be able to answer this question once this is completed.

We found that almost all "oncology centres" have a more than $22 \%$ prevalence of cancer in adnexal masses, whereas this is below $22 \%$ for other centres. ${ }^{2}$ However, we took the view that clinicians will always know their type of centre but might not know the prevalence of cancer in the masses seen in their department.

\section{Competing interests: None declared}

Candido-dos-Reis FJ. Ovarian cancer risk model needs a more meaningful clinical parameter. BMJ 2014;349: g6689.

2 Van Calster B, Van Hoorde K, Valentin L, Testa AC, Fischerova D, Van Holsbeke C, et al; International Ovarian Tumour Analysis (IOTA) Group. Evaluating the risk of ovarian cancer before surgery using the ADNEX model to differentiate between benign, borderline, early and advanced stage invasive, and secondary metastatic tumours: prospective multicentre diagnostic study. BMJ 2014;349:g5920. (15 October.) 